Norbert Kühn

\title{
HERKUNFT UND DIVERSITÄT DER GEHÖLZE IN LANDSCHAFTLICHEN GÄRTEN AM BEISPIEL DER PARKS DER STIFTUNG PREUSSISCHE SCHLÖSSER UND GÄRTEN BERLIN-BRANDENBURG (SPSG)
}

\begin{abstract}
Die Verwendung fremdländischer Gehölze hat in der Gartenkunst eine lange Tradition. Im Staat Preußen entstand durch die Errichtung einer Landesbaumschule 1823 ein europaweit herausragendes Sortiment an Gehölzen. Ein Teil davon wird noch heute im Park von Sanssouci bewahrt, im Jahre 2000 waren es 212 Arten und weitere 116 Sorten. Dies ist ein einmaliger Kulturschatz. Die fremdländischen Bäume konzentrieren sich in den Sonderbereichen der Gärten. Die Parks der Stiftung Preußischer Schlösser und Gärten Berlin-Brandenburg (SPSG) selbst bestehen zu ca. $80 \%$ aus heimischen Gehölzen ${ }^{1}$. Auch ihre Artenzahl ist erstaunlich hoch. Da sich sehr wahrscheinlich die mit diesen Gehölzen verbundenen Lebensgemeinschaften erhalten konnten, sind historische Parks auch für die Vielfalt innerhalb der Ökosysteme von erheblichem Wert. Die Vielfalt an fremdländischen und heimischen Gehölzen und damit verbunden auch die genetische und ökosystemare Diversität können für die Entwicklung von Adaptionsmaßnahmen im Klimawandel von erheblichem Nutzen sein. Gebietseigene Herkünfte haben sich stets in den Gärten erhalten. Fremdländische Arten wurden akklimatisiert und auf ihre Vitalität im Gastland hin selektiert. Sie könnten als Ausgangspunkt auch für die Grüne Infrastruktur der Städte dienen.
\end{abstract}

The use of exotic woody plants in the art of garden design has a long tradition. In the State of Prussia the opening of a regional tree nursery in 1823 resulted in an outstanding range of woody plants across Europe. Some of them are still conserved today in the park of Sanssouci, in 2000 there were 212 species and a further 116 varieties. This constitutes a unique cultural treasure. The exotic trees are concentrated in special sections of the gardens. In the parks of the Prussian Palaces and Gardens Foundation Berlin-Brandenburg (SPSG) around

Im Text werden Maskulinum und Femininum verwendet, wenn es um Personen geht. Gemeint sind grundsätzlich alle Menschen, gleich welcher Geschlechtsidentität sie sich zugehörig fühlen.

1 Heimische Arten: Arten, die ohne menschliche Mithilfe ein Gebiet besiedelt haben oder hier entstanden sind (nach Kowarik 2010). 
$80 \%$ of the woody plants are indigenous ${ }^{2}$. The number of plant species is astonishingly high, too. As the biocenoses linked to these woody plants could very probably be maintained, historic parks are also of considerable value for diversity within the ecosystems. The diversity of exotic and indigenous woody plants and, by extension, the genetic and ecosystem diversity can be of considerable benefit for the development of climate change adaptation measures. Woody plants of native origin have always persisted in the gardens. Exotic species became acclimatised and selected on the basis of their vitality in the host country. They could also serve as the starting point for the green infrastructure of cities.

\section{Gehölzdiversität und Klimawandel}

In den letzten Jahrzehnten verstärkte sich das Interesse an der Biodiversität urbaner Standorte. Neben dem spontanen Grün, neben Straßen und Verkehrswegen und Privat- bzw. Kleingärten hat man sich seit Ende des 20. Jahrhunderts auch den historischen Parks zugewandt. Ziel dieser Untersuchungen waren verwilderte historische Zierpflanzen (»Stinzenpflanzen«) (Bakker/Boeve 1985; Nath 1990; von der Lippe/Kowarik 2006), reliktisch auftretende Arten der ursprünglichen Kultur- und Naturlandschaften (Wulf 1995; Kowarik 1998; Peschel 2000) und in Gärten kultivierte invasive Neophyten (McKinney 2002; Richardson/Rejmánek 2011; Bradley et al. 2012). Um das Ausbreitungsverhalten dieser Arten genauer nachzuvollziehen, wurde die Einführungsgeschichte insbesondere der invasiven Gartenarten genauer aufgearbeitet (Reichard/White 2001; Dehnen-Schmutz et al. 2007; Bradley et al. 2012). Die »normale« kulturbedingte Biodiversität historischer Parks, also ihr Reichtum an fremdländischen Pflanzen, selektierten Kultivaren und Sorten, ist zwar in zahlreichen Inventaren historischer Gärten und Parks niedergelegt, war bislang jedoch kein Schwerpunkt der Forschung.

Die Erhaltung und Erhöhung von Diversität wird als wichtige Möglichkeit gesehen, dem Klimawandel zu begegnen. Besonders im Zusammenhang mit Stadt- und Straßenbäumen wird dies diskutiert (Morgenroth et al. 2016). Dabei wird darauf gedrungen, auch fremdländische Arten zu verwenden (Roloff et al. 2009), sodass beim Ausfall bestimmter Arten und Sorten Alternativen zur Verfügung stehen (Prinzip der Redundanz). Diese Vorgehensweise soll die Resilienz städtischer Ökosysteme fördern. "If `native-only< approaches become incorporated in regional, national or international policy documents or legislation, there is a risk that urban ecosystem resilience will be compromised, particularly for urban areas with extreme environmental conditions which often prevail along roads or in paved areas" (Sjöman et al. 2016, 240). Von anderer Seite wird im Zusammenhang mit der Klimavorsorge aber auch ein stärkerer Rückgriff auf autochthone bzw. gebietseigene Herkünfte gefordert

2 Indigenous species: species that have colonised an area or have emerged here without any human assistance (according to Kowarik 2010). 
(von der Lippe/Kowarik 2014). Sie könnten durch jahrhundertelange Anpassung an Standort und Klimasituation besser geeignet sein als Herkünfte aus anderen Regionen.

Sowohl ein Zurückgreifen auf fremdländische Arten als auch ein Schutz der heimischen Biodiversität kann somit als Adaptionsstrategie gesehen werden, um dem Klimawandel zu begegnen. Herkunft, Vielfalt und die innerartliche genetische Diversifizierung sind somit die entscheidenden Faktoren. Historische Gärten lassen sich diesbezüglich als Versuchsfeld begreifen. In ihnen kommt Unterschiedliches zusammen: heimische und exotische Arten, gärtnerisch selektierte und daher vermutlich standortangepasste Herkünfte und neue Genotypen und Sorten aus dem Handel. Durch die Erforschung dieser seit Jahrhunderten in historischen Parks und Gärten vorhandenen Situation lassen sich einerseits Lösungsansätze für Klimaanpassungsstrategien in städtischen Zusammenhängen liefern, andererseits lässt sich die Resilienz historischer Gärten stärken.

\section{Heimische Gehölze in historischen Parks}

Grundlage für landschaftliche Gärten waren immer die heimischen Gehölze. Sie waren entweder schon auf dem Gelände vorhanden oder wurden aus der näheren Umgebung entnommen, waren also gut erhältlich und somit günstig zu erwerben. Deshalb können heute sogar Pflanzen aus der dem Park vorangegangen Kulturlandschaft zu finden sein. Für den Park von Sanssouci konnte erstmals nachgewiesen werden, dass es dort immer noch Eichen gibt, die aus der Zeit vor der Parkgestaltung stammen (Kühn et al. 2017a). Somit macht es Sinn, Parks auch als mögliche Quelle für gebietseigenes Material anzugeben. »Historische Gärten mit einem alten Gehölzbestand können ein großes Potenzial für die Bewahrung der regionalen genetischen Vielfalt darstellen« (von der Lippe/Kowarik 2014, 53).

Leider ist viel zu wenig über die Plastizität von Baumarten bezüglich wichtiger, den Klimawandel betreffender Umweltfaktoren bekannt (Nicotera et al. 2010). Es wird aber vermutet, dass Populationen, die es über Jahrhunderte vor Ort vermochten, sich an ändernde Klimafaktoren koevolutionär anzupassen, besser geeignet sind, sich auch an kommende Veränderungen anzupassen als Pflanzen fremder Provenienz (von der Lippe/Kowarik 2014). Dies ist zunächst durch die Entstehung indigener Genotypen zu erklären, die auch eine räumliche Differenzierung des Genpools nach sich zog (Jürgens et al. 2007). Dies führte zu einer morphologischen und physiologischen, also phänotypischen Anpassung an die lokalen Bedingungen.

Für eine erfolgreich an die vorherrschenden Umweltbedingungen adaptierte Pflanze spielt allerdings nicht nur die genetische Grundausstattung eine Rolle, sondern auch der Prozess der Anpassung und Optimierung während der Wachstumsphase des jeweiligen Individuums. Diese individuelle Anpassung rückt als Epigenetik zunehmend in den Mittelpunkt der Forschung (Bräutigam et al. 2013). Das bedeutet letztlich, dass nicht nur die lokale Herkunft, sondern auch lokales Aufwachsen Voraussetzungen dafür sind, dass eine erfolgreiche individuelle Standortadaption stattfindet. 
Jenseits dieser genetischen Überlegungen haben Gärtner über Jahrhunderte bei der Vermehrung heimscher Pflanzen immer auf die gesunden und wüchsigen Individuen zurückgegriffen. Man hat somit in den Parks eine gärtnerische Selektion hin zu den vitalen Pflanzen betrieben. Daher kann man davon ausgehen, dass erfolgreich im Park etablierte und möglicherweise auch über mehrere Generationen nachgepflanzte Gehölze eine hohe Standorteignung aufweisen (Kühn et al. 2017 b). Bevor man Pflanzen aus unbekannten Quellen verwendet, könnte man auf diese angepassten bzw. erprobten Formen zurückgreifen.

Da der Pflanzenhandel aber bereits sehr früh einsetzte, sind auch bei heimischen Arten in Parks und Gärten Florenverfälschungen nicht auszuschließen. Sicher ist, dass seit der Aufgabe der parkeigenen Baumschulen (gegen Ende des 19. Jahrhunderts) Material aus länderübergreifend agierenden Großbaumschulen verwendet wurde. So könnte es sich bei Pflanzen geringeren Alters auch um fremde Herkünfte handeln. Auch ist nicht ausgeschlossen, dass Gehölze, die schon mehrere Generationen im Park stehen und sich dort auch generativ weiter ausgebreitet haben, inzwischen als intraspezifische Hybride anzusehen sind.

\section{Fremdländische Arten: Bedeutung ihrer (innerartlichen) Diversität}

Die aus gärtnerischer Kultur stammende Diversität und die dort eingebrachten fremdländischen Arten tragen zur örtlichen Vielfalt entscheidend bei. Import- und kulturbedingte Faktoren eines Pflanzenspektrums werden in der bestehenden Diversitätsdiskussion bislang kaum berücksichtigt. In der Regel zielen Bemühungen um die Erhaltung der Biodiversität immer auf den Schutz heimischer Arten ab - selbst dann, wenn anthropogen entstandene Landschaften wie Parks und Gärten geschützt werden sollen. Die Convention on Biological Diversity (Rio de Janeiro 1992) macht hier keinen Unterschied. In der Definition heißt es: »Biological Diversity means the variability among living organisms from all sources $[\ldots]$ : this includes diversity from all species, between species and of ecosystems « (CBD 1992, 3). Auch kultivierte Arten werden als schützenswert angesehen und als Teil der Evolution anerkannt. »Domesticated or cultivated species means species in which the evolutionary process has been influenced by humans to meet their needs (CBD 1992, 3). Diese gärtnerisch geschaffene Vielfalt ist in Gefahr verloren zu gehen bzw. ist zum Teil auch schon verloren gegangen (Kleinschmit et al. 1995), wie auch eine Untersuchung zu den Gärten von Eduard Petzold nachgewiesen hat (Hendrych/Obdržálek 2014; Richter 2014).

Da man beim Aufsammeln fremdländischer Taxa in ihrem Heimatland nicht auf die innerartliche genetische Vielfalt geachtet hat, so wie das bei forstlichen Samenaufsammlungen heute vorgeschrieben ist (FOVG 2006), ist davon auszugehen, dass das in der Fremde gewonnene Material meist nur einen sehr kleinen Ausschnitt der genetischen Vielfalt der Herkunftspopulationen enthält. Mitunter war man sogar an ganz besonderen Typen interessiert, sodass die Pflanzensammler schon vor Ort eine Selektion bezüglich bevorzugter Eigenschaften (z.B. besonders aufrechter Wuchs, besonders intensive Nadel- bzw. Blattfär- 
bung) vornahmen. Es ist also sehr wahrscheinlich, dass die meisten der nach Deutschland eingeführten fremdländischen Gehölze nur einen Typus mit bestimmten Eigenschaften verkörpern und nicht die ganze genetische Diversität der Art widerspiegeln. Daher könnten einige der fremdländischen und seit Langem in den Parks kultivierten Arten trotz fehlender Sortenbezeichnung eher als Kultivare zu werten sein. Um bei einer Rekonstruktion einer historischen Parkanlage den Denkmalwert zu erhalten, wäre es somit notwendig, nicht nur die jeweilige Art nachzupflanzen, sondern es müsste der damals eingeführte und gebräuchliche Typus erneut verwendet werden. Hierzu besteht noch weiterer Forschungsbedarf.

Es ist anzunehmen, dass zum Ende des 19. Jahrhunderts die Diversität der verwendeten Gehölze ihren Höhepunkt erreicht hatte, wenn auch genaue Zahlen fehlen. Heute geht Kowarik $(2010,85)$ davon aus, dass »etwa 3150 nichteinheimische Gehölzarten « in deutschen Parks und Gärten kultviert werden.

\section{Grundsätzliche Möglichkeiten der Pflanzenherkunft}

Die fortwährende menschliche Einflussnahme lässt in Parkanlagen auf eine sehr komplexe Gemengelage bezüglich Herkunft und genetischer Diversität der Gehölze schließen (siehe Tabelle 1).

\begin{tabular}{|c|c|}
\hline \multirow[t]{3}{*}{ Heimisches Gehölz } & $\begin{array}{l}\text { mit gebietseigener Herkunft, mitunter sogar aus der Zeit vor der } \\
\text { Parkentstehung }\end{array}$ \\
\hline & $\begin{array}{l}\text { mit gebietseigener Herkunft, aber nur als Selektion eine besonderen } \\
\text { Geno- bzw. Phänotyps im Park vorhaben }\end{array}$ \\
\hline & nicht autochthonen Ursprungs, aus dem Handel bezogen \\
\hline \multirow[t]{2}{*}{ Fremdländisches Gehölz } & mit breiter genetischer Amplitude \\
\hline & $\begin{array}{l}\text { bereits bei der Gewinnung im Heimatland vorselektiert und mit einer } \\
\text { geringen Plastizität bezüglich wichtiger Eigenschaften (Frosthärte, } \\
\text { Herbstfärbung, Blütenbildung, Wuchsform) }\end{array}$ \\
\hline \multirow[t]{2}{*}{ Hybrid } & vegetativ weiter vermehrt und damit genetisch identisch gehalten \\
\hline & $\begin{array}{l}\text { aus einem Hybridschwarm, generativ weiter vermehrt und zum Teil auch } \\
\text { immer wieder neu erzeugt }\end{array}$ \\
\hline \multirow[t]{2}{*}{ Sorte } & aus dem Handel, dort generativ vermehrt \\
\hline & aus dem Handel aus vegetativer Vermehrung \\
\hline Typ, Hybrid oder Sorte & $\begin{array}{l}\text { im Park entstanden und dort auch selektiert (parkautochthoner Phäno- } \\
\text { bzw. Genotyp) }\end{array}$ \\
\hline Genetische Mischform & unterschiedliche Herkünfte durch generative Ausbreitung \\
\hline
\end{tabular}

Tabelle 1 Grundsätzliche Möglichkeiten der genetischen Herkunft von Parkgehölzen 
In der Regel werden Gehölzsorten vegetativ vermehrt, was zu genetisch identischen Nachkommen führt. Es gibt aber auch Typen (wie z.B. Säuleneichen, Blutbuchen oder sonstige rotlaubige Gehölze), die bevorzugt generativ vermehrt wurden. Da bei den Sämlingen die gewünschten Eigenschaften unterschiedlich ausgeprägt waren, konnten die besten wiederum ausgelesen werden, sodass letztlich Samensorten entstanden. Abkömmlinge einer solchen Samensorte gelangten bei Bedarf dann wieder in die vegetative Vermehrung.

\section{Die Herkunft der Gehölze in den Gärten der SPSG}

Ab dem 16. Jahrhundert wurden erste Pflanzenkataloge gedruckt und im 17. Jahrhundert entwickelten sich auch in Deutschland erste Handelsgärtnereien (Wimmer 2012), Gehölze waren dabei nur ein Nebenprodukt. Zur gleichen Zeit etablierte John Tradescant der Ältere (1570-1638) einen Pflanzenhandel (Wimmer 2012), der schon Einführungen aus Nordamerika im Sortiment hatte, darunter auch entsprechende Gehölze. So konnte man also ab dieser Zeit Gehölze auch über Handelsgärtnereien beziehen. Obst- und Forstbaumschulen existierten in Deutschland seit dem 17. Jahrhundert (Butenschön et al. 2012), und ab Ende des 18. Jahrhunderts sind Baumschulen für Park- und Ziergehölze nachweisbar (Wimmer 2012). Günther $(1970,7)$ gibt an, dass Friedrich II. für Sanssouci bereits 1746 Eiben, Wacholder und Schwarze Johannisbeeren aus Hinterpommern einführte, später auch »Lärchen, Tannen, Eiben, Kastanien, Linden, Buchen, Rüstern, Ebereschen, Pappeln, Espen, Ahorn und andere Holzarten «. Im gleichen Jahr erhielt er offensichtlich Platanen, Rosskastanien, Tulpenbäume, Scheinakazien, »Cedern und amerikanische Fichten « aus Chelsea (Wimmer 2000, 12). Um 1760 sind Maulbeerbäume aus den Niederlanden und weitere Gehölze aus England bezeugt (Günther 1970, 7). So kamen bereits im 18. Jahrhundert viele Gehölze von Baumschulen aus England und den Niederlanden nach Potsdam und in den Botanischen Garten in Berlin (Wimmer 2012).

Nach einigen kleineren privaten und öffentlichen Vorläufern entstand ab 1795 die erste größere Baumschule im Park von Sanssouci. Auf dieser Fläche »zwischen chinesischer Küche und Freundschaftstempel« standen etwa 180000 Bäume (Wimmer 2000, 13). 1824 erfolgte die Gründung der königlichen Landesbaumschule zu Potsdam in Preußen in Wildpark (Jühlke 1872). Somit musste das Pflanzenmaterial, das man nicht aus der umgebenden Natur entnehmen konnte, nicht teuer von außen angekauft werden. Laut Bethe (1826, 71f.) war es das Ziel »die für Klima und Boden sich eignenden edlen und reichlich zutragenden Obstbäume von allen Gattungen zu billigen Preisen [zu] erhalten«. Aber es sollte auch »zur Ausschmückung der Gärten und Bepflanzung der Wege und wüsten Plätze in den Fluren das Bedürfnis an Waldbäumen und Schmuckgeholz verschiedener Art dort befriedigt werden« (Bethe 1826, 71f.). Ein weiteres Anliegen war das Erproben (Akklimatisieren) ausländischer, bislang nicht gebräuchlicher Gehölze. 1825 wird von 17 amerikanischen Eichenarten berichtet, die sich in der Baumschule befanden. Man interessierte sich 
für diese Pflanzen sowohl aufgrund des Einsatzes im Forst »als auch des Schmuckes wegen für unsere Parks und Gärten« (o. Verf. 1825, 177).

Bereits im Entstehungsjahr standen in der Landesbaumschule, neben zahlreichen Obstbäumen, »50.000 theils amerikanische, theils einheimische zu Alleen und Parkanlagen geeignete Gehölze« (o. Verf. 1825, 72f.). Die Vermehrungsware gewann man durch Aussaat von eigenem oder zugekauftem Saatgut, Stecklinge oder auch Veredelung. Auch Waldbäume wurden ausgegraben und in den Baumschulen aufgeschult. Bei Pflanzenmangel wurden sie allerdings zu dieser Zeit weiter auch direkt der Natur entnommen, was als Missstand angeprangert wurde (Jühlke 1872).

Zur Mitte des 19. Jahrhunderts ließ das Angebot kaum noch Wünsche offen: »In den Deutschen Gehölzbaumschulen befinden sich, außer der Masse von Rosenspielarten und den sogenannten Coniferen (noch seltenen, nicht acclimatisirten, meistens zum Nadelholz gehörenden, immergrünen Holzarten), über 1500 verschiedene Arten von Bäumen und Gesträuchen, darunter allein ungefähr 250 einheimische Arten, sämmtlich bestimmt, in Gärten und Parkanlagen und bei Verschönerungen gepflanzt zu werden « (Jäger 1857, 127). Offensichtlich hatte die Landesbaumschule ein besonders breites Sortiment. Laut Wimmer $(2000,15)$ »bezeichnete der Breslauer Botaniker Rudolf Göppert das Sortiment der Landesbaumschule als umfangreichste und vollständigste Sammlung Europas«.

Ende des 19. Jahrhunderts übernahmen große private Baumschulen den Markt. Aus Handelsgärtnereien mit meist gemischtem Sortiment hatten sich marktbeherrschende Unternehmen wie die Späth'sche Baumschule entwickelt. Öffentliche Einrichtungen konnten mit dieser Entwicklung nicht mehr mithalten. Das führte zur Schließung der Landesbaumschulen. Die preußische Landesbaumschule in Potsdam ging 1893 in Konkurs und fortan bezog man die Gehölze fast ausschließlich aus dem Handel. Eine eigene Anzucht von Gehölzen wurde in geringem Umfange weitergeführt, auch nach der Umwandlung der Hofverwaltungen in staatliche Schlösserverwaltungen gab es in den historischen Anlagen noch vereinzelt parkeigene Baumschulen. Im Laufe der Zeit wurden immer mehr Flächen aufgegeben, bis schließlich nur unbedeutende Reste übrig blieben (Wacker 2017).

\section{Diversität des Baumbestandes in landschaftlichen Anlagen der SPSG}

\section{Vielfalt der Altbäume}

Für die Abschätzung der Diversität der Gehölze in den Anlagen der SPSG kann auf unterschiedliche Quellen zurückgegriffen werden. Die Gartenverwaltung führt ein Baumkataster, um die Vitalität der Altbäume regelmäßig zu dokumentieren. Für das Projekt »Zukunftsweisender Umgang mit der Gehölzvegetation historischer Gärten in Zeiten des Klimawandels« wurden diese Daten für fünf Parks (Charlottenburg, Glienicke, Neuer Garten, Sanssouci, Pfaueninsel) ausgewertet (siehe Tabelle 2). Den größten Bestand an Altbäumen und 


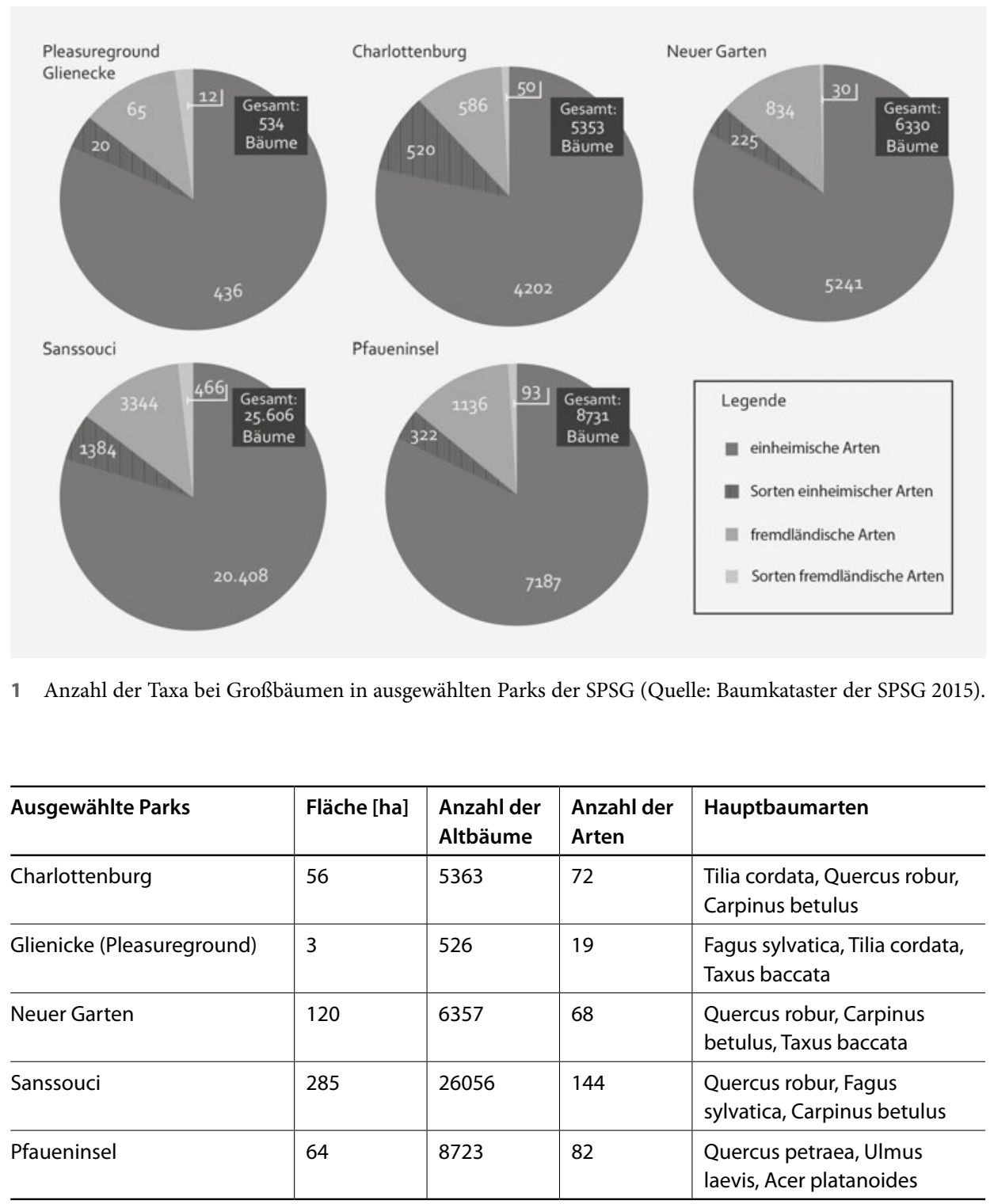

Tabelle 2 Untersuchte Parks in der SPSG und ihr Altbaumbestand (Quelle: Bestandsaufnahme SPSG 2015)

auch an Gehölzarten weist Sanssouci auf, was natürlich auch der Flächengröße geschuldet ist. Junge Bäume, Sträucher und Klettergehölze sind in diesen Daten nicht erfasst.

Ermittelt man die Anzahl der Taxa (siehe Abb. 1), zählt man also die Anzahl der Arten, Unterarten, Varietäten und Sorten, so sind es in Sanssouci 240. Die heimischen Arten sind 


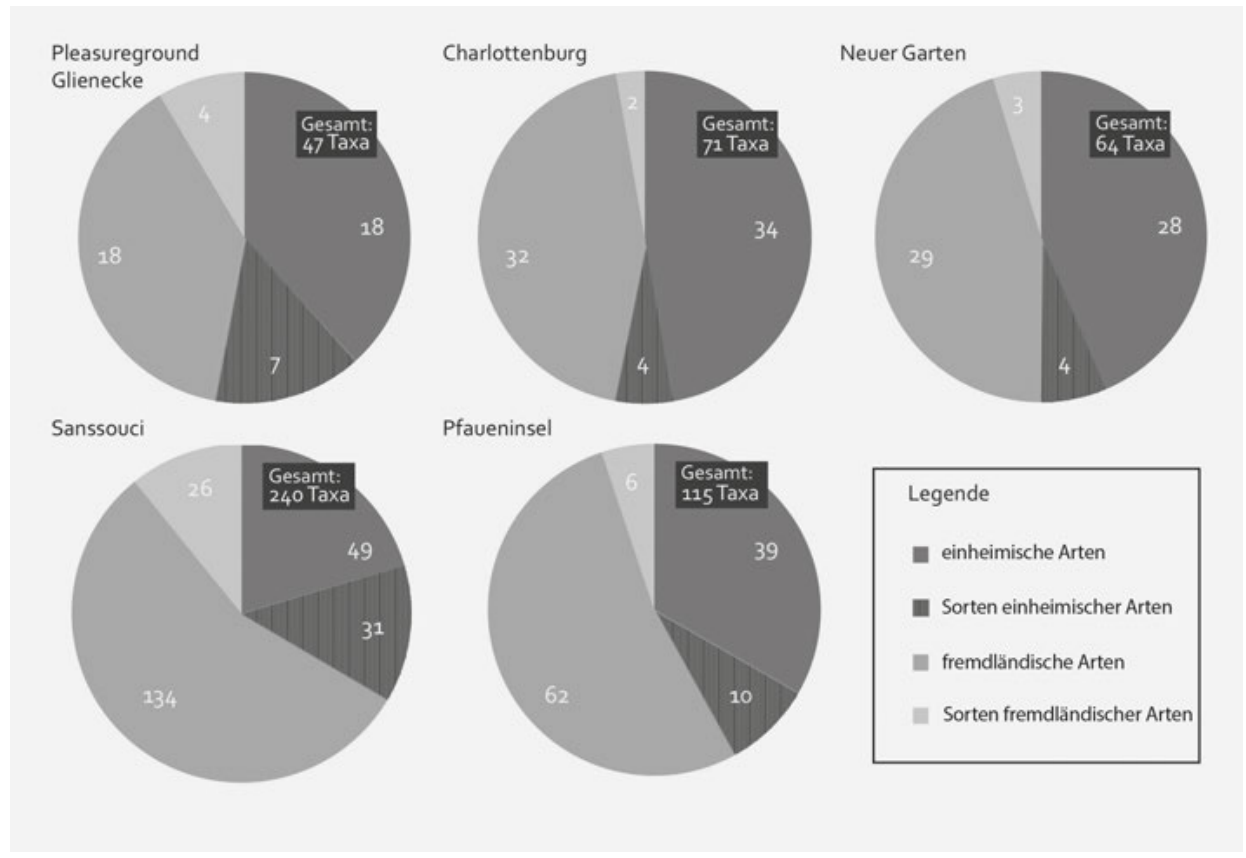

2 Anzahl der Großbäume in ausgewählten Parks der SPSG, aufgeteilt nach ihrer Herkunft (Quelle: Baumkataster der SPSG 2015).

prozentual in der Minderheit. Nur $20 \%$ aller Taxa sind heimisch, dazu kommen noch einmal $13 \%$ mit Sorten heimischer Arten. $56 \%$ sind fremdländisch und davon gibt es noch einmal $11 \%$ mit Sorten fremdländischer Arten. In den anderen Parks sind die Zahlen ausgeglichener. Das lässt sich einfach erklären: Die Anzahl der heimischen Arten ist beschränkt, 49 Baumarten dürften schon fast das gesamte, überhaupt in Deutschland verfügbare Spektrum darstellen. ${ }^{3}$ Mithin können nur noch fremdländische Arten hinzukommen; je größer der Park ist, umso mehr fremdländische Arten kann er also enthalten.

Betrachtet man jedoch die Individuen der Großbäume in den Parks, so zeigt sich ein ganz anderes Bild (siehe Abb. 2). Einheimische sind wesentlich häufiger, sie treten bestandsbildend in allen waldartigen Beständen auf. Fremdländische sind dagegen wesentlich seltener und vor allem in Sonderbereichen wie den Pleasuregrounds oder Themengärten zu finden. Überraschend sind hier die durchweg ähnlichen Prozentzahlen: Obwohl es sich bei Glienicke nur um den Pleasureground handelt, Pfaueninsel, Neuer Garten und Charlottenburg ganz unterschiedliche Entstehungszeiten haben und Sanssouci aus mehreren, auch einzeln ansprechbaren Gartenteilen besteht, besitzen sie doch grundsätzlich zu ca. $80 \%$

3 Zum Vergleich: für die Stadtfläche von Berlin werden insgesamt 29 vorkommende heimische Bäume angegeben, siehe Kowarik et al. 2013, 120. 


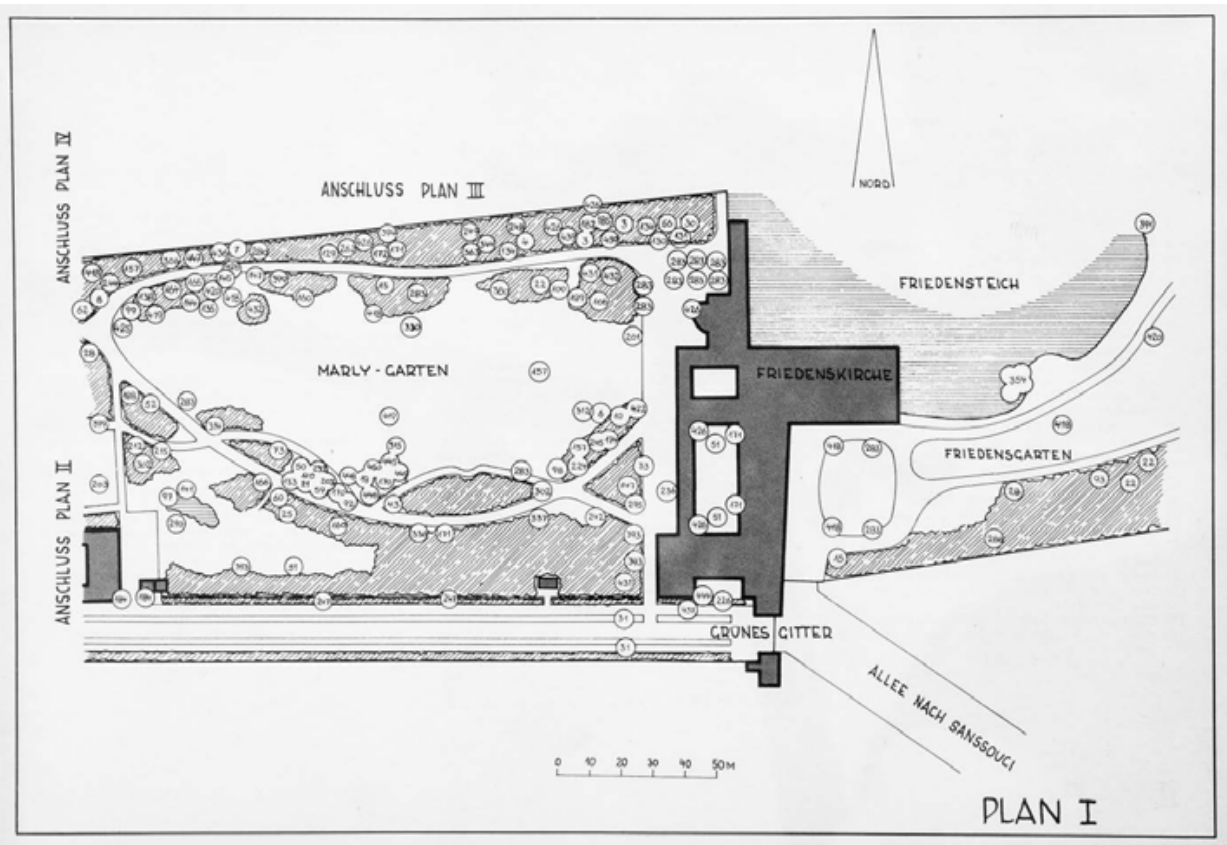

3 Verteilung der Gehölze im Marly-Garten. Die Nummern geben besonders herausragende Gehölze an (Günther 1981, 59).

heimische Bäume. Die Fremdländischen liegen bei 11 bis $13 \%$, und die Sorten fremdländischer Arten spielen zumindest bei den Großbäumen bezogen auf die Gesamtindividuen in einem Park kaum eine Rolle. Der Eindruck, dass die Landschaftsparks überwiegend aus nichtheimischen Arten und Sorten bestehen, täuscht somit. Sie nehmen bei den Großbäumen nur ca. $10 \%$ ein, fallen aber wahrscheinlich beim Betrachter stärker ins Gewicht, da sie optisch hervortreten.

\section{Gesamtbestand der Gehölze in Sanssouci}

Aktuelle Listen des Gesamtbestandes der Gehölze in den Parks der Stiftung gibt es nicht. Es existieren jedoch Baumführer für den Park von Sanssouci aus den Jahren 1970, 1981 und 2000 (Günther 1970; Günther 1981; SPSG 2000), sodass ein Vergleich der Entwicklung in diesen Jahren möglich ist.

In diesen Führern werden bestimmte Bereiche einzeln erfasst. Es handelt sich um besonders gehölzreiche Flächen, wie den Marlygarten, den Nordischen und Sizilianischen Garten und den Bereich um das Schloss Charlottenhof. Für den Marlygarten (ca. 4 ha) werden 111 Pflanzen mit Nummern vermerkt (siehe Abb. 3). Die sie umgebenden, wohl in 


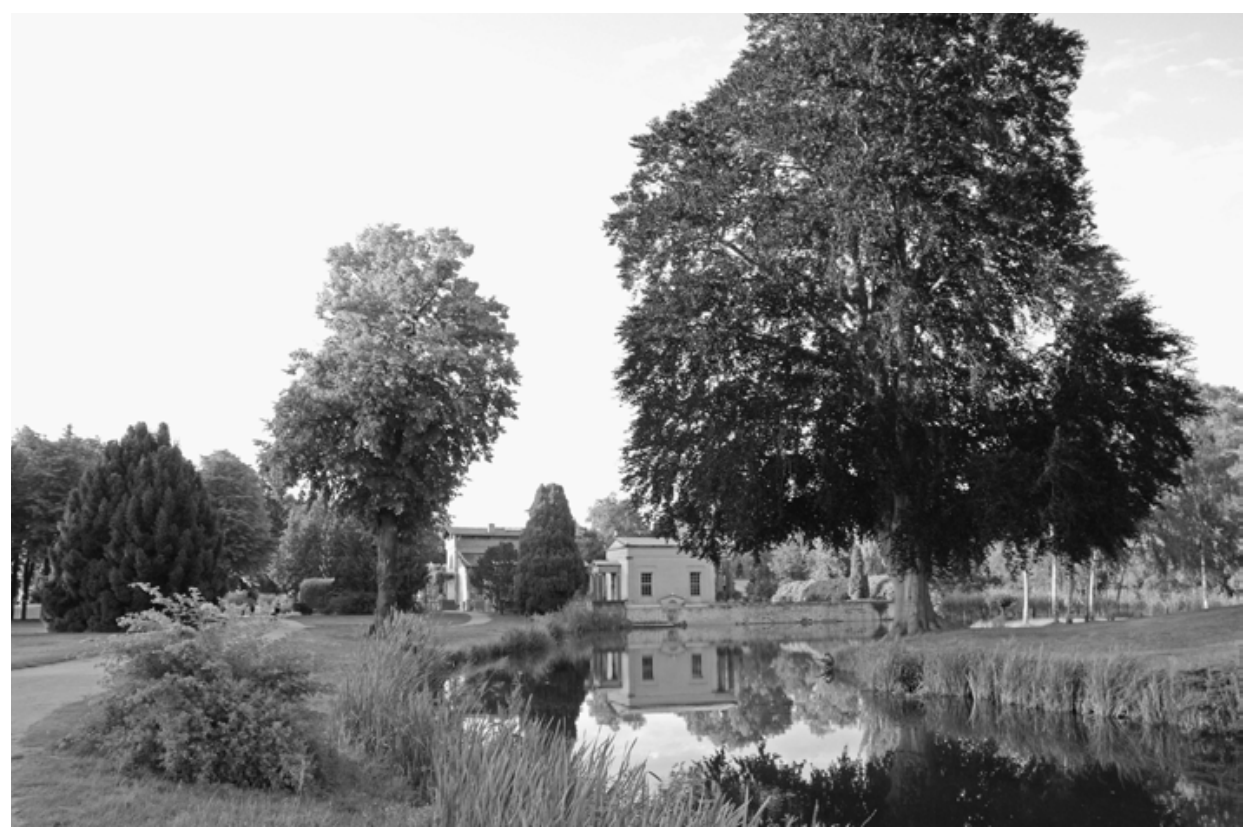

4 Die Römischen Bäder im Park von Sanssouci - ein Bereich mit einem besonders hohen Reichtum an Gehölzen.

der Regel einheimischen Gehölze sind nicht verzeichnet, sodass man von einer noch höheren Gesamtzahl ausgehen muss. Im Marlygarten kommen somit etwa 30 Taxa pro Hektar vor - im gesamten Park sind es im Durchschnitt 1,3. Sieht man sich die Werte der Taxa bei den Großbäumen für Sanssouci pro Hektar an, sind es nur 0,2. Die Pleasuregrounds sind also eindeutig die Zentren der Diversität (Abb. 4).

Die Listen der Gehölzführer zählen wesentlich mehr Taxa auf als die der Altbaumkartierungen. Es sind 374 (1980), 454 (1981) und 432 (2000) Arten, Unterarten, Varietäten und Sorten. Zu den Laub- und Nadelbäumen kommen Kletterpflanzen und v.a. Sträucher. Die Sträucher machen etwa ein Drittel aller erhobenen Taxa aus und tragen damit ganz entscheidend zur Gehölzdiversität in den Parks der Stiftung bei (siehe Abb. 5).

Die Anzahl der heimischen Gehölzarten liegt bei 57 (SPSG 2000). Sie ist damit nur wenig geringer, als die bei Kowarik et al. $(2013,120)$ für ganz Berlin angegebene (dort 67). Das heißt auf der Fläche von Sanssouci (285 ha) gibt es in etwa so viele Arten an heimischen Gehölzen wie in ganz Berlin (ca. 90000 ha).

Vergleicht man Baumkataster und Gehölzführer für Sanssouci, so unterscheiden sie sich in Bezug auf die heimischen Gehölze kaum (von 49 auf 57 bzw. bei den Sorten von 31 auf 49). Jedoch gibt der Gehölzführer deutlich mehr nichtheimische Arten (212) und auch Sorten (126) an. Offensichtlich ist bei den Wuchsformen, die bei den Baumkartierungen nicht vertreten sind (Sträucher, Zwergsträucher, Klettergehölze), die Anzahl der fremdlän- 


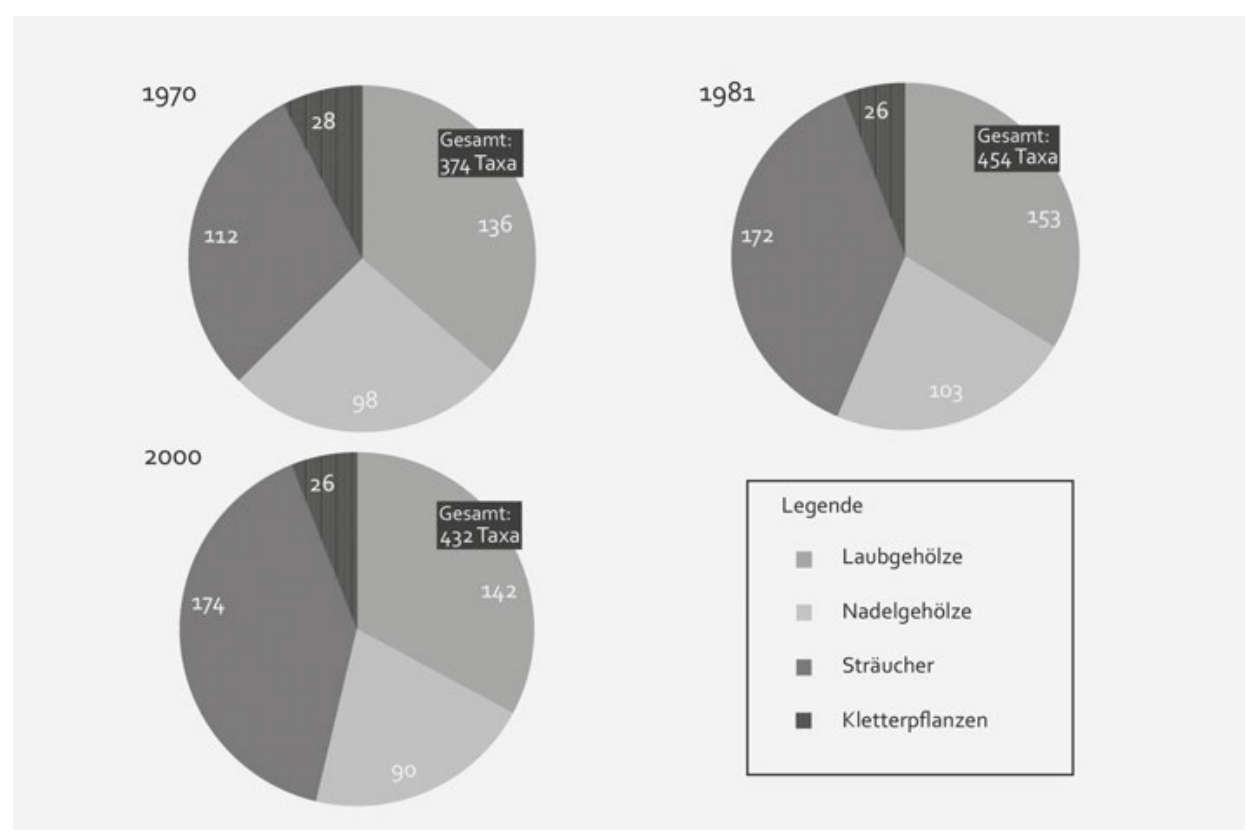

5 Anzahl der Taxa im Park von Sanssouci 1970, 1991 und 2000 (Quelle: Gehölzführer der SPSG [Günther 1970; 1991; SPSG 2000]).

dischen Arten und ihrer Sorten besonders hoch. Deshalb verschiebt sich das Verhältnis im Gehölzführer insgesamt zu den fremdländischen Arten und Sorten.

Veränderung der Gehölzflora in den Jahren 1970 bis 2000

Überraschende Ergebnisse zeigt der Vergleich der Gehölzführer aus den Jahren 1970, 1981 und 2000 (siehe Abb. 6). Eigentlich sollte man davon ausgehen, dass in historischen Gärten die verwendeten Taxa immer wieder dieselben sind. So würde man es im Sinne der Charta von Florenz auch erwarten (»Artikel 9; [...] Die Authentizität eines historischen Gartens beruht sowohl auf dem Plan und der räumlichen Konzeption seiner verschiedenen Partien als auch auf der schmückenden Ausstattung, der Pflanzenauswahl und den Baumaterialien « (Charta von Florenz, 1981, Übersetzung Erika Schmidt)). Ein Vergleich der Gehölzführer aus den unterschiedlichen Jahren zeigt jedoch, dass die verwendeten Arten und Sorten erheblichen Schwankungen unterworfen sind. So kommen 1981125 Taxa hinzu, die es 1970 noch nicht gab, im Jahr 2000 sind es 59. Es verschwinden jedoch auch welche aus den Gärten, 1981 45, im Jahr 2000 sogar 81. Auch wenn diese Schwankungen mitunter auf neue Erkenntnisse (Korrektur falsch bestimmter Pflanzen z.B.) zurückzuführen sind, zeigt es doch, dass das Inventar historischer Gärten nie still steht - auch 


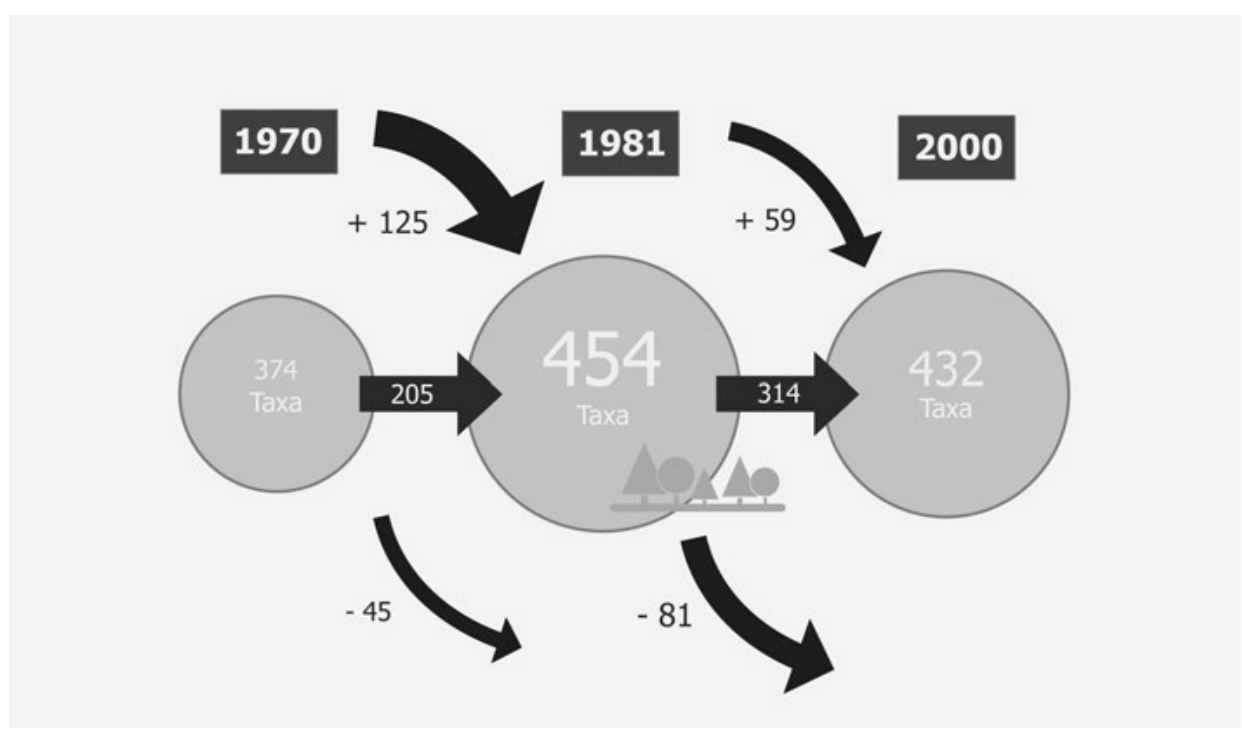

6 Veränderung im Artenspektrum der Gehölze von Sanssouci von 1970 bis 2000 (Quelle: Drei Gehölzführer der SPSG [Günther 1970; Günther 1991; SPSG 2000]).

wenn es sich um gartendenkmalpflegerisch vorbildlich entwickelte Anlagen wie die der SPSG handelt. Wie lässt sich das erklären?

Viele der Pflanzen sind nur in wenigen Stückzahlen vorhanden - und können daher leicht verschwinden. Extreme klimatische Ereignisse (z.B. Spätfröste) oder Krankheiten (Buchsbaumzünsler) können die Populationen bestimmter Taxa gefährden. Neue Erkenntnisse führen dazu, dass man andere Arten an eine bestimmte Stelle setzt. Auch werden durch weiterentwickelte Vorgehensweisen zur Erhaltung und Pflege bestimmte Maßnahmen eingeführt - und später wieder aufgegeben (z.B. die DDR-zeitlich beliebten Fruchtwechsel bei Bäumen).

Letztlich betont diese Erkenntnis aber nur, dass historische Gärten keine statischen Kunstwerke sind. Der Wechsel sogar des Arten- und Sorteninventars bei Gehölzen ist Teil ihrer Konzeption. Deshalb braucht es auch immer kreative Köpfe, um diesen Wechsel zu begleiten und Entscheidungen zu treffen, die einen Fortbestand im Geiste gewährleisten und nicht nur im Sinne einmal (fest)gesetzter Listen.

\section{Diskussion}

Es bleibt festzuhalten, dass die Parks der SPSG hauptsächlich aus heimischen Gehölzarten bestehen (zu ca. $80 \%$ aller Altbäume). Auch ihre Biodiversität ist erstaunlich hoch: Allein in Sanssouci gibt es ca. 57 heimische Gehölze und damit fast so viele wie in ganz Berlin. Es 


\begin{tabular}{|c|c|c|c|}
\hline Kategorie & Eignung & Anzahl & Arten \\
\hline Kategorie 1.1 & $\begin{array}{l}\text { Bzl. Trockentoleranz } \\
\text { und Winterhärte } \\
\text { sehr geeignet }\end{array}$ & 17 & $\begin{array}{l}\text { Acer campestre, A. negundo, A. tataricum, Alnus } \\
\text { incana, Buxus sempervirens, Crataegus x lavallei, } \\
\text { C. communis, Ostrya carpinifolia, Pinus aristata, } \\
\text { P. nigra var. austriaca, P. sylvestris, Populus tremula, } \\
\text { Prunus avium, P. mahaleb, Rhamnus catharticus, } \\
\text { Robinia luxurians, R. pseudoacacia }\end{array}$ \\
\hline Kategorie 1.2 & $\begin{array}{l}\text { Bzl. Trockentoleranz } \\
\text { sehr geeignet, } \\
\text { bzl. Winterhärte } \\
\text { geeignet }\end{array}$ & 22 & $\begin{array}{l}\text { Acer monspessulanum, Ailanthus altissima, Carya } \\
\text { alba, Cedrus libani, Celtis occidentalis, Eleagnus } \\
\text { commutata, Ginkgo biloba, Gleditsia triacanthos, } \\
\text { Maclura pomifera, Morus alba, Pinus ponderosa, } \\
\text { Platanus x hispanica, Populus alba, Prunus cerasifera, } \\
\text { Pyrus salicifolia, Quercus cerris, Q. frainetto, Q. libani, } \\
\text { Q. macranthera, Q. prinus, Sophora japonica, Tilia } \\
\text { tomentosa }\end{array}$ \\
\hline Kategorie 2.1 & $\begin{array}{l}\text { Bzl. Trockentoleranz } \\
\text { geeignet, bzl. } \\
\text { Winterhärte sehr } \\
\text { geeignet }\end{array}$ & 14 & $\begin{array}{l}\text { Aesculus x carnea, Betula pendula, Carpinus } \\
\text { betulus, Crataegus crus-galli, C. monogyna, Fraxinus } \\
\text { pennsylvanica, Picea omorica, Pinus mugo var. } \\
\text { mughus, Populus x berolinensis, Quercus robur, Salix } \\
\text { caprea, Tilia intermedia, T. cordata, T. x euchlora }\end{array}$ \\
\hline Kategorie 2.2 & $\begin{array}{l}\text { Bze. Trockentoleranz } \\
\text { geeignet, bzl. } \\
\text { Winterhärte } \\
\text { geeignet }\end{array}$ & 14 & $\begin{array}{l}\text { Carya ovata, Castanea sativa, Corylus colurna, } \\
\text { Fraxinus excelsior, Gymnocladus dioicus, Laburnum } \\
\text { anagyroides, Liquidambar styraciflua, Mespilus } \\
\text { germanica, Phellodendron amurense, Robinia hispida, } \\
\text { Quercus imbricata, Q. palustris, Q. petraea, Q. rubra }\end{array}$ \\
\hline
\end{tabular}

Tabelle 3 Gehölze im Park von Sanssouci (Daten: Gehölzführer der SPSG 2000), die nach Roloff als Zukunftsbaumarten im Sinne der KlimaArtenMatrix (KLAM) gelten können (Roloff 2013)

ist daher davon auszugehen, dass sich mit diesen Gehölzen verbundene Lebensgemeinschaften aufbauen konnten. In diesem Sinne sind die Gärten von ganz erheblichem Wert.

Aber auch fremdländische Bäume kommen vielfach vor, meist in geringen bis sehr geringen Stückzahlen. Sie konzentrieren sich in den Sonderbereichen der Gärten und ihre Verwendung hat eine lange gartenkünstlerische Tradition. Im Staat Preußen wurde sie insbesondere ab dem Ende des 18. Jahrhunderts sehr gefördert. Durch die Errichtung der Landesbaumschule entstand ein europaweit herausragendes Sortiment an Gehölzen. Ein Teil davon wird noch heute im Park von Sanssouci bewahrt, im Jahre 2000 waren es 212 Arten und weitere 116 Sorten.

Der Vergleich der Gehölzführer von 1970, 1981 und 2000 zeigt, dass das Sortiment nicht statisch ist. Pflanzen kommen hinzu und verschwinden wieder. Dies lässt darauf schließen, dass auch ein gewisser Turn-Over stattfindet und man zumindest bei den seltenen Arten immer wieder auf Ersatz von außen angewiesen ist. Nicht zuletzt deshalb ist nur schwer einzuschätzen, von welcher Herkunft die im Park stehenden Gehölze tatsächlich sind. Möglichkeiten gibt es viele (siehe Tabelle 1). Um die genaue Herkunft nachzuweisen, 
bedarf es außer einem eingehenden Quellenstudium auch dendrochronologischer und genetischer Untersuchungen.

Diversität wird als eine wichtige Strategie zur Klimaanpassung gesehen. Die genauere Untersuchung der in den Parks der Stiftung prosperierenden - oder der wenig vitalen und abgängigen - Bäume könnte Aufschlüsse darüber liefern, inwieweit die Gehölze für die Zukunft geeignet erscheinen. Roloff (2013) gibt in seiner KLimaArtenMatrix (KLAM) Gehölze an, die bezüglich steigender Temperatur und zunehmender Trockenheit eine hohe Toleranz aufweisen. Vergleicht man diese Aufstellung der Zukunftsbäume mit den in den Parks vorkommenden Arten, so erkennt man, dass viele dort schon seit langem gepflanzt und etabliert sind (siehe Tabelle 3). Die Erkenntnisse, die in den Parks gewonnen werden, können so auch für die Verwendung von Bäumen außerhalb dieser Anlagen von Bedeutung sein.

\section{Literaturverzeichnis}

Bakker, Piet; Boeve, Evert (1985): Stinsenplanten. Zutphen: Uitgeverij terra.

Bethany, A Bradley; Blumenthal, Dana M.; Early, Regan; Grosholz, Edwin D.; Lawler, Joshua J.; Miller, Luke P.; Sorte, Cascade J. B.; D’Antonio, Carla M.; Diez, Jeffrey M.; Dukes, Jeffrey S.; Ibanez, Ines; Bradley, Julian D. Olden (2012): Global change, global trade, and the next wave of plant invasions. In: Frontiers in Ecology and the Environment 10.1, 20-28.

Bethe, Carl Gottlieb (1826): Rede des Direktors des Gartenbau-Vereins am Jahresfeste den 20. Juni 1824. In: Verhandlungen des Vereins zur Beförderung des Gartenbaues in den Königlich Preußischen Staaten. Bd. 2. Berlin, 70-72.

Bräutigam, Katharina; Vining, Kelly J.; Lafon-Placette, Clément; Fossdal, Carl G.; Mirouze, Marie; Gutiérrez, Marcos José; Fluch, Silvia; Fernández Fraga, Mario; Guevara M., Ángeles; Abarca, Dolores; Johnsen, Øystein; Maury, Stéphane; Strauss, Steven H.; Campbell, Malcolm M.; Rohde, Antje; Díaz-Sala, Carmen; Cervera, María-Teresa (2013): Epigenetic regulation of adaptive responses of forest tree species to the environment. In: Ecology and evolution 3.2, 399-415.

Butenschön, Sylvia; Palm, Heike; Beck, Jens (2012): Frühe Baumschulen - Produktion für das Schöne und das Nützliche. In: Frühe Baumschulen in Deutschland. Hg. von Silvia Butenschön. Berlin: TU Berlin, 7-13.

CBD (1992): Convention on Biological Diversity. Adopted in Rio De Janeiro, 5 June 1992. https://treaties.un.org/ doc/Treaties/1992/06/19920605\%2008-44\%20PM/Ch_XXVII_08p.pdf (15.08.2017)

Dehnen-Schmutz, Katharina; Touza, Julia; Perrings, Charles; Williamson, Mark (2007): A century of the ornamental plant trade and its impact on invasion success. In: Diversity and Distributions 13, 527-534.

Günther, Harri (1970): Gehölze in den Gärten von Sanssouci. Dendrologischer Führer. Potsdam: Eigenverlag der SPSG.

Günther, Harri $\left(1981^{2}\right)$ : Gehölze in den Gärten von Sanssouci. Dendrologischer Führer. Potsdam: Eigenverlag der Staatlichen Schlösser und Gärten Potsdam-Sanssouci.

Hoemann, Reinhold (1908): Die Einfachheit in der Gartenkunst. In: Die Gartenkunst 10.9, 147-154.

Hendrych, Jan; Obdržálek, Jan (2014): Historic Trees and Avenues. In: Wiedergeburt von Baumgiganten. Hg. Von Claudius Wecke und Katrin Weber. Berlin: be.bra wissenschaft verlag GmbH, 199-214.

Jäger, Hermann (1857): Die Verwendung der Pflanzen in der Gartenkunst. Oder: Gehölz, Blumen und Rasen. Ein künstlerischer Führer bei der Anlage und Unterhaltung von Landschafts- und Blumengärten, für Gärtner, Guts- und Gartenbesitzer. Leipzig: Wöller.

Jühlke, Ferdinand (1872): Die Königliche Landesbaumschule und Gärtnerlehranstalt zu Potsdam geschichtliche Darstellung ihrer Gründung, Wirksamkeit und Resultate, nebst Cultur-Beiträgen. Berlin: Wiegandt und Hempel.

Jürgens, Anna; Seitz, Birgit; Kowarik, Ingo (2007): Genetic differentiation of Rosa canina (L.) at regional and continental scales. In: Plant systematics and evolution 269.1-2, 39-53. 
Kleinschmit, Jörg; Begemann, Frank; Hammer, K. (1995): Erhaltung pflanzengenetischer Ressourcen in der Land-und Forstwirtschaft.

Kowarik, Ingo (1998): Historische Kulturlandschaften als Objekte des Naturschutzes. In: Naturschutz und Denkmalpflege. Hg. von Ingo Kowarik, Erika Schmidt und Birgit Sigel. Zürich: VDL Hochschulverlag AG, 111-139.

Kowarik, Ingo $\left(2010^{2}\right)$ : Biologische Invasionen. Neophyten und Neozoen in Mitteleuropa. Stuttgart: Ulmer.

Kowarik, Ingo; Lippe, Moritz; Cierjacks, Arne (2013): Prevalence of alien versus native species of woody plants in Berlin differs between habitats and at different scales. In: Preslia 85.2, 113-132.

Kühn, Norbert; Fischer, Sarah; Gillner, Sten; Rohde, Michael; Schmidt-Wiegand, Antje; Seeliger, Anja (2017a): Zukunftsweisender Umgang mit der Gehölzvegetation historischer Gärten in Zeiten des Klimawandels. In: Jahrbuch der Baumpflege 21, 155-173.

Kühn, Norbert; Gillner, Sten; Schmidt-Wiegand, Antje (Hg.) (2017b): Gehölze in historischen Gärten im Klimawandel. Berlin: TU Berlin, L\&U, 131.

McKinney, Michael L. (2002). Influence of settlement time, human population, park shape and age, visitation and roads on the number of alien plant species in protected areas in the USA. In: Diversity and distributions 8.6, 311-318.

Morgenroth, Justin; Östberg, Johan; Konijnendijk van den Bosch, Cecil; Nielsen, Anders B.; Hauer, Richard J.; Sjöman, Hendrik; Chen, Wang; Jansson, Märit (2016): Urban tree diversity - Taking stock and looking ahead. In: Urban forestry \& urban greening 15, 1-5.

Nath, Martina (1990): Historische Pflanzenverwendung in Landschaftsgärten. Auswertung für den Artenschutz. Worms: Wernersche Verlagsgesellschaft.

Nicotra, Adrienne B.; Atkin, Owen K.; Bonser, Stephen P.; Davidson, Amy Michelle; Finnegan, E. Jean; Mathesius, Ulrike; Poot, Pieter; Purogganan, Michael D.; Richards, Christina L.; Valladares, Fernando; van Kleunen, Mark (2010): Plant phenotypic plasticity in a changing climate. In: Trends in plant science $15.12,684-692$.

O. Verf. (1825): XXXV. Auszug aus der Verhandlung aufgenommen in der fünf und zwanzigsten Sitzung des Vereins am 9. Januar 1825. In: Verhandlungen des Vereins zur Beförderung des Gartenbaues im Preuß. Staate. Vierte Lieferung. Verhandlungen 2. Band, 177.

Peschel, Tim (2000): Vegetationskundliche Untersuchungen der Wiesen- und Rasengesellschaften historischer Gärten in Potsdam. Stuttgart: Ibidem Verlag.

Reichard, Sarah Hayden; White, Peter (2001): Horticulture as a pathway of invasive plant introductions in the United States. In: BioScience 51.2, 103-113.

Richardson, David M.; Rejmánek, Marcel (2011): Trees and shrubs as invasive alien species - a global review. In: Diversity and distributions 17.5, 788-809.

Richter, Susanne (2014): Gehölzvielfalt in den Gärten von Eduard Petzold. In: Wiedergeburt von Baumgiganten. Hg. von Claudius Wecke und Katrin Weber. Berlin-Brandenburg: be.bra wissenschaft verlag, 183-197.

Roloff, Andreas (2013): Bäume in der Stadt. Stuttgart: Ulmer-Verlag.

Roloff, Andreas; Korn, Sandra; Gillner, Sten (2009). The climate-species-matrix to select treespecies for urban habitats considering climate change. In: Urban Forestry \& Urban Greening 8.4, 295-308.

Sjöman, Henrik; Morgenroth, Justin; Sjöman, Johanna; Deak; Sæbø, Arne; Kowarik, Ingo (2016): Diversification of the urban forest - Can we afford to exclude exotic tree species? In: Urban Forestry \& Urban Greening 18, 237-241.

Stiftung Preußischer Schlösser und Gärten Berlin-Brandenburg (Hg.) (2000³): Gehölze in den Gärten von Sanssouci. Dendrologischer Führer. Potsdam: Eigenverlag.

Verhandlungen des Vereins zur Beförderung des Gartenbaues in den Königlich Preußischen Staaten. Elfter Band. Berlin. Auf Kosten des Vereins. 1835. IV. Ueber Akklimatisirung der Gewächse. Vom Herrn Geheimen Medizinal-Rath, Professor Dr. Link.

Von der Lippe, Moritz; Kowarik, Ingo (2006): „Die Vorgärten und Anlagen sind die naturbelebte Ergänzung der Wohnung «. In: Der Garten - ein Ort des Wandels. Hg. von Eric A. de Jong, Erika Schmidt und Brigit Sigel. Zürich: VDF, 101-116.

Von der Lippe, Moritz; Kowarik, Ingo (2014): Warum weiter mit dem Originalbaum? In: Wiedergeburt von Baumgiganten. Hg. Claudius Wecke und Katrin Weber. Berlin-Brandenburg: be.bra wissenschaft verlag, 49-60. 
Wacker, Jörg (2017): Überlegungen zur lokalen Anzucht von Gehölzen in den Gärten der SPSG. In: Gehölze in historischen Gärten im Klimawandel. Hg. von Norbert Kühn, Sten Gillner und Antje Schmidt-Wiegand. TU Berlin, L\&U 131, 226-232.

Wimmer, Clemens Alexander (2000): Beschreibung der Gehölze. In: Gehölze in den Gärten von Sanssouci. Hg. von der Generaldirektion der SPSG. Potsdam. Eigenverlag, 21-134.

Wimmer, Clemens Alexander (2012): Zur Entstehung der Baumschulen in Deutschland. In: Frühe Baumschulen in Deutschland. Hg. von Silva Butenschön. Berlin: TU Berlin, 15-44.

Wulf, Matthias (1995): Historisch alte Wälder als Orientierungshilfe zur Waldvermehrung. In: LÖBF-Mitteilungen 1995.4, 62-71.

\section{Bildnachweis}

1, 2, 4-6 Norbert Kühn; 3 Stiftung Preußische Schlösser und Gärten Berlin-Brandenburg. 